Agenda Internacional

Año XXIV N 35, 2017, pp. 51-66

ISSN 1027-6750

\title{
La nueva administración estadounidense y su impacto en la relación bilateral con México
}

\author{
María del Pilar Ostos Cetina*
}

\section{RESUMEN}

El propósito de este ensayo consiste en reflexionar acerca del estado que guardan las actuales relaciones entre México y Estados Unidos a partir del ascenso del mandatario Donald Trump, lo cual ha tenido un impacto mediático significativo, que a su vez ha incidido en la postura que han asumido los mandatarios de ambos países; lo que ha alentado las reivindicaciones de tipo nacionalista y la tendencia hacia el proteccionismo económico, tal como se evidencia en el caso estadounidense a través del contenido del discurso y la praxis del America First. $\mathrm{Al}$ mismo tiempo, se han generado varias expectativas de cambio o continuidad en lo que se refiere a la atención de los temas prioritarios de la agenda bilateral, comenzando por los efectos en la ampliación del muro de "contención» fronteriza ante flujo masivo de inmigrantes, el devenir del Tratado de Libre Comercio de América del Norte (TLCAN), además de los aspectos que refieren a las cuestiones de seguridad nacional a partir del combate a los carteles de la droga y la neutralización de crimen organizado, cuyos efectos trascienden de lo bilateral al ámbito de los asuntos hemisféricos.

Palabras clave: Estados Unidos, México, nacionalismo, proteccionismo, América First, TLCAN, muro de contención fronteriza, migración, seguridad nacional.

\section{The New US Administration and its Impact on the Bilateral Relationship with Mexico}

\section{Abstract}

The purpose of this essay is to reflect on the state of current relations between Mexico and the United States after the rise of President Donald Trump, which has had a significant media impact, which in turn has influenced the posture which have assumed the leaders of both countries; which has encouraged nationalist claims and the trend toward economic protectionism, as evidenced in the American case through the content of America First's discourse

* Docente e investigadora del Centro de Estudios Superiores Navales de la Universidad Naval, Secretaría de Marina Armada de México. Correo electrónico: mpostos@hotmail.com 
and praxis. At the same time, there have been several expectations of change or continuity with regard to addressing the priority issues on the bilateral agenda, beginning with the effects on the expansion of the border "containment" wall in the face of massive influx of immigrants, The development of the North American Free Trade Agreement (NAFTA), as well as aspects related to national security issues from the fight against drug cartels and the neutralization of organized crime, whose effects go beyond the bilateral to hemispheric issues. Keywords: United States, Mexico, nationalism, protectionism, America First, NAFTA, border retaining wall, migration, national security. 


\section{Antecedentes de las relaciones bilaterales México-Estados Unidos}

Tras conocerse el impacto mediático que genera por sí mismo Donald Trump, primero en su condición de empresario de la construcción y del entretenimiento, y posteriormente a partir de su postulación para ocupar la presidencia de Estados Unidos, han surgido numerosas voces a favor y en contra de la postura que asumió este nuevo mandatario, al proponer como parte de su discurso de campańa y ya en el ejercicio de su cargo, una renovación profunda y contundente del quehacer político tanto en los aspectos domésticos, entre los que se incluye a México como parte sustancial de la América del Norte, y también en aquellos asuntos de carácter global que, desde la perspectiva del actual mandatario, implica según sus propias palabras, la apertura de «un nuevo capítulo de la grandeza de Estados Unidos» (BBC Mundo, 2017).

En consonancia con este tipo de objetivos que pretende lograr la nueva administración al frente de la Casa Blanca, el tema de la relación bilateral con México se torna prioritario, tomando en consideración las condiciones de vecindad geográfica y hasta geopolíticas en la que convergen ambas naciones en cuestión. Para ello, resulta indispensable hacer un breve balance de estas relaciones que, desde antes, particularmente en pleno siglo XIX, al calor de procesos paralelos encaminados a la consolidación como Estados-nacionales, darían lugar a un primer momento de fricción y conflicto que derivó, después de lo militar, en la firma del Tratado Guadalupe Hidalgo en el año de 1848.

Un hecho singular que, a la postre, le permitiría al "coloso del norte» expandirse tras obtener la soberanía de los actuales estados de California, Nevada, Utah, Nuevo México y Texas. Además de alcanzar el predominio sobre Arizona, Colorado, Wyoming, Kansas y Oklahoma; estableciendo una nueva cartografía para referenciar la ampliación de los límites físicos que la dirigencia política de Estados Unidos se había propuesto siguiendo el curso del río Bravo y que, analizado esto a la luz de los hechos actuales, nos sitúa frente al mismo escenario geográfico donde convergen una multiplicidad de hechos y factores, entre ellos: el flujo masivo de inmigrantes ilegales y el fenómeno delincuencial asociado al narcotráfico, lo que sin duda, exacerba las tensiones entre ambas naciones a partir de cuestiones de carácter fronterizo.

Efectivamente, esa nueva delimitación física de ambos territorios supuso para México la pérdida de 1,3 millones de $\mathrm{km}^{2}$ —equivalente a casi la mitad de su territorio- $\mathrm{y}$ cerca del 1\% de su población. De los 100000 mexicanos afectados por la partición, aproximadamente 75000 decidieron quedarse en lo que para ese momento pasaría a ser de la jurisdicción de Estados Unidos, y a su vez derivó en la creación de una frontera que se prolongaría a través de 3200 kilómetros de longitud, tal como sigue vigente hasta nuestros días (Pastor Gómez, 2016, p. 3). 
Así, el resguardo de este perímetro de seguridad le implica a Estados Unidos destinar importantes recursos físicos y materiales, con miras a garantizar la vigilancia y el control de aquellos puntos de acceso, lo que con el correr de los años le ha obligado a más de un gobierno en este país a «blindar» su frontera con mayor equipamiento tecnológico y reforzar este límite a través de la construcción de una valla física, lo que nos remite a la idea de un muro diseñado por tramos, que comprende hasta ahora 1050 kilómetros de construcción y dispone de un total de 40 puntos de cruces legales. A pesar de este monitoreo permanente, siguen existiendo áreas que resultan del interés de quienes se empeñan, bajo su propio riesgo, atravesar el territorio mexicano en dirección a Estados Unidos como parte de la búsqueda de lo que el historiador James Truslow Adams, en 1931, describió como el «sueño americano» (Palm y Stikkers, 2017, p. 57) ${ }^{1}$.

Cabe destacar que, dentro de esta misma problemática, el conjunto de los territorios que en el pasado habían pertenecido a México, progresivamente han sido ocupados por población, esencialmente de origen mexicano, lo que a la fecha y en términos numéricos se aproxima a la cifra de 34,6 millones de personas de un total de 55,4 millones de hispanos que residen actualmente en Estados Unidos, y son quienes conforman el 17\% de la población total de dicho país (BBC Mundo, 2016a; Presidencia de la República, 2016).

En ese mismo contexto, la postura del presidente Trump ha sido la de señalar enfáticamente que, algunos de los inconvenientes de orden interno que afectan a su nación, principalmente en materia delictiva, radica en aquellos 11,3 millones de inmigrantes indocumentados, entre quienes 5,6 millones son de origen mexicano y de los cuales, haciendo algo de comparación con respecto a los dos mandatos anteriores con el presidente Barack Obama, se logró en su momento la deportación de por lo menos 2,5 millones de personas acusados de permanecer ilegalmente en suelo estadounidense. Por su parte, la actual administración de gobierno se ha propuesto expulsar superar dicha cifra, expulsando a todos aquellos inmigrantes que, además de indocumentados, tengan antecedentes judiciales o pertenezcan a agrupaciones delictivas o dedicadas al tráfico de drogas (BBC Mundo, 2016b).

Otro de los aspectos a destacar en medio de la condición asimétrica que caracteriza las relaciones entre México y Estados Unidos, se puede comprender a la luz del intercambio comercial. En ese sentido, y tras la puesta en marcha del TLCAN en el año de 1994, Estados Unidos se ha convertido en el primer socio comercial de México,

\footnotetext{
$\mathrm{Al}$ respecto, Adams describe tal expresión en su libro The Epic of America, «como ese sueño sobre una tierra donde la vida sea mejor, más rica y más plena para cada uno de los hombres, con oportunidades para todos de acuerdo a sus capacidades y logros».
} 
captando el 80,2\% de sus exportaciones. Un aspecto singular hoy en día, no solo para el gobierno mexicano, lo es también para el conjunto de los empresarios nacionales e inversionistas foráneos que, desde México, se han visto beneficiados del envío constante y expedito de mercancías con destino al vecino país del norte.

De este modo, México se erige como uno de los socios comerciales más importantes para el mercado estadounidense, ocupando el segundo lugar en las exportaciones de Estados Unidos, después de Canadá y el tercero en importaciones, luego de la presencia china y canadiense, lo que a últimas fechas ha generado un comercio bilateral de más de 532 mil millones de dólares anuales (Presidencia de la República, 2016). Al tiempo que convierte a Estados Unidos en el primer inversionista extranjero en México que alcanza un total de 57,7\%, según datos recientes de la revista Forbes.

En lo que respecta a los temas de defensa y seguridad, un aspecto crucial que modificó el esquema de las relaciones bilaterales en esta materia entre los dos países, se dio precisamente durante la presidencia del mandatario mexicano Vicente Fox (20002006), cuya intensión de suscribir un tratado migratorio con sus contrapartes en Estados Unidos tuvo escaso margen de apoyo. Sin embargo, lo que se convirtió en un aspecto prioritario para Washington fue el impulso que desde entonces enfatizó a la política antidrogas a nivel continental, comenzando con la implementación del llamado Plan Colombia (1998), convertida en una estrategia que se extendió hasta México, lo que le implicó a los estamentos castrenses mexicanos, además de otras instituciones del Estado, efectuar una serie de ajustes para estar acorde a los lineamientos de la política de seguridad nacional estadounidense a través de lo pactado en la llamada Iniciativa Mérida (2008), conocida también como Plan Mérida o Plan México, cuyo alcance en cuestiones de antinarcóticos y de otros aspectos afín, se extendieron al resto del área de Centroamérica (Pastor, 2016, p. 5).

Considerando esta serie de antecedentes que definen, enmarcan y enfatizan el carácter disímil en la relación México-Estados Unidos, aunado a las expectativas que aún sigue generando la presencia mediática del actual mandatario estadounidense, ya sea a través de las redes sociales y más concretamente, asumiendo decisiones como las que se han dado a conocer en el curso de poco más de cien días de iniciada su administración de gobierno, comenzando por la desvinculación de Estados Unidos de varios mecanismos de orden multilateral, destacándose su salida del Tratado de Asociación Transpacífico (TTP), seguido de su autoexclusión del Acuerdo de París sobre cambio climático. Esto sin dejar de considerar, la reciente determinación del gobierno por aquietar los términos de lo pactado por su antecesor en lo que respecta al descongelamiento de las relaciones con la isla de Cuba, entre tanto no se concreten las acciones debidas que conlleve a la celebración de elecciones libres y se brinden las garantías de libertad entre los presos políticos en la isla. 


\section{Los fundamentos del nacionalismo de Trump y su incidencia en la relación con México}

De acuerdo a lo anterior, los movimientos ideados por el establishment estadounidense que encabeza la presidencia de Trump, con miras a devolver la grandeza de Estados Unidos en medio del actual entorno global, conlleva una serie de expectativas para México en lo que respecta, al devenir de la ampliación del muro de contención fronteriza, la finalización o continuidad del TLCAN, seguido de las especulaciones que aún se ciernen sobre la viabilidad y el financiamiento de los proyectos de cooperación en materia de defensa y seguridad, todos establecidos con anterioridad entre las instituciones homólogas de ambos países.

Así, una manera de comprender más a detalle los lineamientos que enfatiza la actual administración de gobierno de Estados Unidos y su visión con respecto a la implementación de criterios nacionalistas en su toma de decisiones, se retoman de lo expresado por el presidente Trump (2015) en su libro: Crippled America. How to make America Great Again, en cuyo contenido hace alusión a una serie de apreciaciones de tipo personal con alcance político, advirtiendo sobre una apremiante necesidad de cambio para su nación, tras observa una cierta «inmovilidad» y un rezago que constrińe e impide un posicionamiento real y efectivo de Estados Unidos en el contexto que ofrece el nuevo siglo.

En ese sentido, el presidente Trump se ha propuesto, siguiendo con lo expresado en su libro, diseñar toda una apología que conlleve de nueva cuenta a «hacer grande a Estados Unidos. Esto significa, «restaurar ese sentido de dignidad para la Casa Blanca, y para nuestro país en general», según sus propias afirmaciones. Al tiempo que enfatiza en que precisamente, la figura del presidente de Estados Unidos, representa a «la persona más poderosa del mundo, por ser el portavoz para la democracia y la libertad» (p. 247).

A partir de tales conjeturas, la actual administración de gobierno se propone retomar los fundamentos doctrinarios que en su momento formularon los "Padres Fundadores» (founding fathers), lo que a su vez implica, considerar las bases de lo que en palabras de Max Weber alude al expresar los contenidos de la «ética protestante y al espíritu del capitalismo», siendo este en el —gran motor ideológico- de una dirigencia política que se configura en términos académicos en lo que actualmente se denomina una "corporatocracia», encargada de proponer y llevar a la praxis los principios básicos de la democracia, la libertad comercial y las medidas que favorezcan el orden y la seguridad a todo el orbe internacional.

En ese sentido, y retomando la percepción del mandatario Trump, se trata de recomenzar con la «obra» de modernización del país o lo que en su primer discurso ante 
el congreso decidió bautizar como la formulación de un «Programa de reconstrucción nacional». Adjudicando quizás un sello muy personal, mediante el cual el jefe del ejecutivo pretende poner a prueba su experiencia en el terreno de los negocios y de la construcción inmobiliaria, a una escala mayor en la que se relancen, de nueva cuenta, los intereses prioritarios de su nación con un alcance mundial.

En tal sentido, nos encontramos frente a esa especie de autoevaluación de los tratados, acuerdos y demás planes ejecutados por su antecesor, con la finalidad de identificar las limitaciones y los retos que enfrenta la propia nación para acrecentar su autosuficiencia y a su vez, garantizar el pleno derecho de mantener su hegemonía global en la contienda frente a otras naciones, con la intensión de fortalecer sus capacidades productivas; para lo cual se identificó como parte sustancial del problema que ha llevado a un mermado desarrollo de la nación estadounidense, el posicionamiento de dos de las más destacas economías emergentes: México y China.

\section{México en el «blanco» de las acusaciones del presidente Trump}

Bajo esa condición, ambas naciones pasaron a convertirse antes y después de la contienda electoral para el cargo de presidente de Estados Unidos, en el «blanco» perfecto de las acusaciones de Trump. Además de considerarse, particularmente a México como la principal motivación y por ende, la más clara justificación de aquellas primeras órdenes ejecutivas que diera a conocer el presidente en su primer día al frente de los destinos de la Casa Blanca.

Coincidiendo con lo anterior, una revisión puntual del contenido del discurso del America First, más allá de expresar una alta dosis de nacionalismo, expone de manera clara y contundente lo que para la dirigencia política estadounidense bien se puede considerar como una de sus principales amenazas, lo que en palabras del presidente Trump se expresó en los siguientes términos: «Juntos vamos a determinar el curso de Estados Unidos y del mundo en los años por venir. ...(Por ello), debemos de proteger nuestras frontera de otros países que producen y destruyen nuestros empleos. Protegernos nos va a permitir prosperar en grande y de forma poderosa» (www.whitehouse.gov).

Así, sin haberse mencionado en el discurso del America First el nombre de México, ciertamente este aparece de manera implícita, particularmente cuando se refiere al control de los puestos de trabajo en manos de la población que procede de fuera, lo que se atribuye en gran medida, al paso descontrolado de inmigrantes a través de la extensa frontera que comparten estas dos naciones vecinas. Con estos argumentos, que más parecen la descripción puntual del origen de la amenaza que se cierne sobre Estados Unidos, el presidente Trump se ha propuesto desde que era candidato electo, 
rediseñar y ampliar la cobertura de un amplio muro fronterizo. Proponiendo que este se convierta en una barrera artificial de contención, capaz de amortiguar a la población inmigrante proveniente no solo del país vecino del lado sur, sino del resto del continente y de otras latitudes del planeta.

$\mathrm{Al}$ respecto, el presidente Trump afirmó, «la primera cosa que necesitamos hacer es asegurar nuestra frontera sur y nosotros necesitamos hacerlo ahora mismo. Tenemos que detener esa avalancha, y la mejor manera de hacerlo es construyendo un muro. ...La gente mala no solo viene de México. Ellos vienen de Centro y Sudamérica, y probablemente de Medio Oriente» (Trump, 2015, pp. 48-55).

De ahí que una de las primeras órdenes ejecutivas que firmó el presidente en pleno ejercicio de sus funciones de gobierno, consistió en gestionar la construcción del muro calculado en 20000 millones de dólares, lo que en su opinión debería ser costeado por México, considerada la nación-problema. Así, lo que en un principio se convirtió en un tema del discurso - sin mucho sentido- durante la campaña de candidato republicano a la presidencia de Estados Unidos, paulatinamente fue tomando mayor impulso y atrajo un mayor número de seguidores, coincidentes con esta iniciativa de contener a toda costa, incluso con un muro, el flujo masivo de inmigrantes, dispuestos a ocupar los puestos de trabajo principalmente de las clases media y baja estadounidenses.

En ese mismo período de campaña, se presentó como un hecho poco recurrente dentro de la política interna de Estados Unidos, la visita del candidato republicano, Donald Trump por invitación expresa del presidente Peña Nieto, a la sede de Palacio Nacional, el pasado 31 de agosto de 2016. Se trató de un encuentro, aparentemente amistoso, propicio para establecer un diálogo moderado con respecto a la iniciativa latente de construcción del muro y el delicado tema de mantener con vida el TLCAN. Al final de la rueda de prensa que ofrecieron ambos mandatarios a los medios de comunicación, la nota principal se presentó cuando el candidato republicano escribió a sus seguidores de Twitter que, a pesar del encuentro, «México will pay for the Wall» (México pagará por el muro). Lo que de inmediato generó la respuesta del mandatario mexicano, Enrique Peńa Nieto, declarando de manera puntual en su cuenta de Twitter que, «México jamás pagará por el muro».

A partir de entonces, los pronunciamientos entre estos dos personajes políticos no cesaron. Por el contrario, tomaron cada vez mayor fuerza a través de distintos medios, incluso después de la toma de posesión del presidente estadounidense, cuando Trump declaró otra vez por Twitter que, «Estados Unidos tiene un déficit comercial de 60 billones de dólares con México. Ha sido un acuerdo unilateral desde el inicio del TLCAN con un número masivo de empresas y puestos de trabajo perdidos. 
Si México no está dispuesto a pagar por la pared necesaria, sería mejor cancelar la próxima reunión (prevista con el Enrique Peña Nieto para el 31 de enero de 2017, en la ciudad de Washington)». En respuesta, el mandatario mexicano informó también por la misma red social: «Esta mañana hemos informado a la Casa Blanca que no asistiré a la reunión de trabajo programada para el próximo martes con el @POTUS» (BBC Mundo, 2017b).

Guiados por la controversia que desde un principio generó la propuesta de ampliación del muro, los niveles de tensión suscitados por parte de la opinión pública mexicana alcanzaron la molestia y un halo de incertidumbre entre diferentes sectores del ámbito político, académico, empresarial, militar y de otros, cuya percepción se tornaba alarmista, avizorando una inminente salida de capitales, presiones sobre la divisa mexicana y una pérdida, sin precedentes, de puestos de trabajo a todo lo largo y ancho del territorio mexicano. Lo anterior daría lugar a una atmosfera altamente negativa en México, que a pesar de ello, comenzó a tornarse favorable a quienes le apostaron por promover la «unidad nacional» y cerrar filas alrededor de la figura presidencial, siendo esta una estrategia para resistir cualquier embate previsto desde Washington (Alcántara, 2017).

Bajo ese mismo contexto, el gobierno de Enrique Peña Nieto formuló cinco principios para encarar la postura de «choque», promovida por la administración de Donald Trump, teniendo como imperativo: 1) fortalecer los criterios de la soberanía nacional para fomentar un diálogo entre países soberanos, 2) Respeto al Estado de Derecho, es decir, respeto a las leyes de México y de Estados Unidos, 3) Establecer una visión constructiva y propositiva para la búsqueda de soluciones creativas con el propósito de ganar-ganar, 4) Fomentar la integración de Norteamérica con mayor dinamismo y competitividad conjunta, 5) Promover la negociación integral con el objetivo llevar a la mesa de negociación todos los temas (Presidencia de la República, 2017).

En consonancia con estos principios, la estrategia del gobierno mexicano consistió y sigue siendo la de mantener abiertos los canales del diálogo formal con las instituciones homologas en Estados Unidos, independientemente de las opiniones coyunturales que a través de redes sociales y por medio de discursos emitiera el presidente Trump. De ahí que con el correr de los meses, el ánimo de confrontación que reinaba entre los jefes de Estado de los dos países, se trasladará a una conversación más fructífera y de trabajo constante entre los pares que se dedican dentro de la administración pública a los asuntos internos, las respectivas cancillerías, las áreas dedicadas al comercio y hacienda, seguido de aquellas que atienden las cuestiones de la seguridad y la defensa nacional, además del trabajo que corresponde a las respectivas embajadas. 


\section{4. ¿Fin o renegociación del TLCAN?}

En medio de este barullo de exaltaciones de carácter nacionalista, intercambio de mensajes a través de las redes sociales entre los presidentes Trump y Peńa, seguido de la insistencia de licitar prontamente para confirmar la construcción del muro fronterizo, una carta que aún está por definirse y que cobra mucho interés entre el empresariado mexicano, es sin lugar a dudas, la salvaguarda o renegociación del TLCAN.

Es claro que en la visión del presidente Trump, se trata de un bad deal para su país. Y lo reafirma aún más cuando advierte, «México ha hecho polvo» a Estados Unidos en materia comercial, según lo muestran los últimos datos estadísticos en los que la balanza comercial se inclina a favor de México con una ventaja de 60000 millones de dólares entre enero y noviembre de 2016. Entre tanto, y con respecto a China, considerado el otro «blanco» de las acusaciones de la reciente administración de gobierno de Estados Unidos, el déficit comercial se ubicó en 319282 millones de dólares, una cifra cinco veces mayor al dato referido para México (González, 2017).

Cabe señalar que, resultados comerciales como los antes descritos, conllevan a un análisis más puntual y preciso de los hechos, al advertir que

[...] no significa que México inunde a Estados Unidos de productos, sino que las empresas de ambos países trabajan en conjunto al producir diversos productos que hacen a la región de América del Norte competitiva. (De este modo), y tras la entrada en vigor del TLCAN (desde 1994), la industria manufacturera de México recibió mayores inversiones y empleó mayor mano de obra, mientras que Estados Unidos tuvo un mayor impulso a todo lo que da el sector servicios (González, 2017).

Es por ello que, el marco que ofrece el TLCAN ha permitido el diseño de una cadena de valor integrada, establecida de manera conjunta entre México y Estados Unidos, lo que ofrece diversas ventajas en términos de precios, favoreciendo en gran medida al consumidor estadounidense en el marco de este esquema de producción. Entre tanto, el caso de China resulta muy distinto, ya que la producción de cada uno de los productos que exporta hacia el mercado estadounidense se elabora en sus propias plantas y con mano de obra local, empleando escasamente un $4 \%$ de insumos provenientes de Estados Unidos, lo que se diferencia ampliamente del modelo establecido con respecto a México.

Frente a este panorama, la decisión del ejecutivo estadounidense a pocos días de inicio del mandato de Trump, incidió de tal manera que varios sus corporativos en México, comenzando por la empresa Ford, tuvieran que desistir de avanzar con sus planes como era la colocación de una planta de ensamblaje calculada en 1600 millones de dólares en el estado de San Luís Potosí. A cambio de retirar sus inversiones en 
México, la empresa automotriz obtuvo la promesa de obtener una rebaja sustancial en el cobro de sus impuestos para operar en Estados Unidos.

A todo esto, el empresario mexicano Carlos Slim, considerado el cuarto hombre más rico del mundo, expresó también su punto de vista entorno a lo que varios analistas calificaban como una crisis, sin precedentes, en la relación México- Estados Unidos. $\mathrm{Al}$ respecto, Slim argumentó que tras su encuentro en diciembre con Trump, observó que «no es un terminator, es un negotiator. No hay que ser catastrofistas. Esperemos que como vayan pasando los días, vaya amainando su hiperactividad» (Hernández, 2017).

Entre tanto, sugirió que esta crisis puede ser más bien una oportunidad de negocios para México, por lo que resulta necesario volcarse sustancialmente al desarrollo de la economía interna. Para lo cual sugirió que «hay que poner énfasis en la inversión nacional y en los programas de infraestructura, vivienda, reconversión urbana y comunicaciones». Y con respecto al muro, Slim explicó que «la mejor barda es invertir en México, fortalecer la seguridad jurídica, la Ley de transparencia, el sistema nacional anticorrupción, bajar el gasto corriente, eficientizar el gasto público y ofrecer empleos a los mexicanos» (Hernández, 2017).

Mientras avanzaban los días tras la toma de posesión de Trump en el poder, y de que, siguiendo a Slim, «amaina la hiperactividad» del presidente estadounidense en lo que respecta a las decisiones combativas frente a México, dicho vaticinio se cumplía en medio del nuevo orden de prioridades que asumía la Casa Blanca tras los hechos de Siria y Medio Oriente, el involucramiento de Rusia en los asuntos domésticos de Estados Unidos, el cerco a Qatar por parte de las naciones árabes, seguido de los efectos que generó en la opinión pública internacional la salida de Estados Unidos de la COP París, sin descuidar la relevancia de los temas con Corea del Norte y por ende con China en la región de Asía Pacífico, solo por citar algunos de los temas que encabezan la agenda de prioridades en Washington. Así, en medio de esta serie de coyunturas, el gobierno mexicano se mantuvo firme y convencido de continuar su labor de "cabildeo» con las oficinas paralelas que el gobierno estadounidense ha designado para analizar los contenidos que darán certidumbre a las reuniones del próximo mes de octubre acerca de la continuidad, renegociación o desaparición del TLCAN.

Obviamente que el costo de una negociación favorable a los intereses trazados por México y por ende, a las pretensiones hegemónicas de Estados Unidos y de Canadá, implica asumir desde ahora responsabilidades y un listado de encomiendas a los cuales Estado mexicano ha tenido que ceder abrir un espacio, tal como se analizará más adelante, por el simple hecho de pertenecer al esquema geopolítico de la América del Norte, cuyo radio de acción se prolonga y trasciende a toda Centroamérica y la región del mar Caribe. 


\section{La cooperación en seguridad México-Estados Unidos: la «defensa por capas»}

Retomando los aspectos centrales del informe Mexico: Background and U.S Relations, elaborado por el servicio de investigación del Congreso de Estados Unidos, destaca que desde que se inició la Iniciativa Mérida a partir de 2008 y hasta 2016, Estados Unidos ha invertido más de US\$2500 millones en este tipo de planes, con la finalidad de combatir a grupos criminales y del narcotráfico, pero también a fortalecer las bases de lo que se conoce como el Programa Frontera Sur.

Precisamente, la puesta en marcha de este tipo de programas como Frontera Sur y las recientes coordinaciones en el llamado Triángulo Norte (integrado por Guatemala, Honduras y El Salvador), que incluyen la participación «operativa y logística» de México a partir de lo que vendría a ser el avance a partir de su frontera sur, se convierte a últimas fechas, en ese «as» sobre la mesa de negociaciones mediante el cual la dirigencia política mexicana pretende mantener con vida al TLCAN. Por su parte, los intereses de Estados Unidos en esta administración de gobierno se siguen manifestando a través de la importancia que reviste la implementación de la política de seguridad hemisférica, la cual desde la perspectiva del actual secretario de Seguridad Nacional, siendo en el pasado el encargado de dirigir la línea de tareas asignadas al Comando Sur, en cuya opinión resulta más que pertinente avanzar en la implementación del esquema de «defensa en capas», lo cual atiende de momento al blindaje que se requiere en atención a la seguridad fronteriza de Estados Unidos (www.elhorizonte.mx).

Esa propuesta de la "defensa en capas" que viene de la iniciativa del secretario de Seguridad Nacional de Estados Unidos, se vinculada de manera estrecha con los resultados de la reciente Conferencia sobre Seguridad y Prosperidad de Centroamérica efectuada durante los días 15 y 16 de junio en la Universidad Internacional de Miami, en la que México y Estados Unidos fungieron como coanfitriones. A dicha conferencia asistieron, los representantes de la delegación mexicana encabezada por el titular de la Secretaria de Gobernación, Miguel Ángel Gutiérrez Chong; el secretario de Relaciones Exteriores, Luis Videgaray Caso y el encargado de la jefatura de Hacienda, José Antonio Meade. Por parte del gobierno de Estados Unidos asistieron, el vicepresidente, Michael Pence, acompańado del secretario de Estado, Rex Tillerson; el secretario de Seguridad Nacional, John F. Kelly; además del secretario del Tesoro, Steven Mnuchin.

Junto a ellos, estuvieron presentes los mandatarios de Guatemala, Jimmy Morales, el presidente de Honduras, Juan Orlando Hernández y el vicepresidente de El Salvador, Oscar Ortíz, entre otros representantes de gobierno de países vecinos como Nicaragua, Panamá y Colombia; además de altos representantes del Fondo Monetario Internacional (FMI), el Banco Mundial (BM) y el propio titular del 
Banco Interamericano de Desarrollo (BID), Luis Alberto Moreno, quien en esta ocasión manifestó el serio "compromiso" del presidente Donald Trump de seguir auspiciando el Plan de la Alianza para la Prosperidad del Triángulo del Norte, en esta ocasión con una oferta más amplia de oportunidades económicas, destinada a dar mayor impuso al capital humano y a mejorar la seguridad ciudadana, todo esto con la intensión primordial de desalentar a toda costa los flujos masivos de inmigrantes con rumbo a los Estados Unidos (www.nodal.am).

Previo a esta reunión celebrada recientemente en Miami, en el mes de abril tuvo lugar en Cozumel (México) otra reunión en la que México y Estados Unidos participaron también como coanfitriones de la misma. Se trató de la $\mathrm{V}$ Conferencia de Seguridad en Centroamérica (CENTSEC), un encuentro que según el Pentágono fue de "cuatro estrellas" por el alto rango de los asistentes a este evento, de cierta manera histórico, por ser el primero en el que ambos países fungían como coanfitriones; pero además, ejemplar en términos de lo que significa el afianzamiento de las relaciones entre los dos estamentos castrenses de los países en cuestión (Hernández Borbolla, 2017).

$Y$ no es en vano que, pese a que en principio se especuló sobre una crisis «inminente» en esta relación bilateral México-Estados Unidos tras el arribo de Trump al poder, lo cierto es que con el avance de los meses, los hechos han demostrado que sin que desaparezcan la - mutua e histórica desconfianza - entre ambas naciones, la importancia de mantener abiertos los canales de diálogo entre los representantes de las instituciones homologas entre ambos países, incluyendo la permanencia de los embajadores de los respectivos países en sus lugares de trabajo, se convierten en una muestra clara de la «madurez política» con la cual se ha pretendido solventar esta relación entre vecinos contiguos.

Otra muestra del trabajo en conjunto entre México y Estados Unidos, se explica con la inauguración de las labores de trabajo de la Asamblea General de la OEA, a celebrarse en la ciudad de Cancún, Quintana Roo, encargada de sesionar los 19 y 21 del junio del año en curso. Al respecto, el canciller mexicano Luis Videgaray señaló que su país «reafirma el compromiso con el multilateralismo y con la OEA, siendo estas herramientas esenciales para enfrentar los grandes retos de la agenda global y regional» (García, 2017).

A su vez, el canciller Videgaray señaló que, "los países de América conforman una región que ha sido pionera en el multilateralismo, que trabajan unidos por la seguridad hemisférica, la consolidación de la democracia representativa, el irrestricto respeto a los derechos humanos y el impulso al desarrollo» (García, 2017), lo cual se convierte en los temas fundamentales del debate de la OEA, pero a su vez, tiene como telón de fondo un tema «coyuntural» en la región como es el devenir de Venezuela. 
En ese contexto, México ha tenido que, en los últimos meses, asumir también el compromiso de liderar una postura firme y directa encabezada, de nueva cuenta por su canciller, Luis Videgaray, quien en diferentes foros ha condenado las acciones antidemocráticas del gobierno venezolano de Nicolás Maduro. Entre tanto, su homóloga venezolana, Delcy Rodríguez, ha respondido a este tipo de declaraciones, señalando que «el país más peligroso del mundo (refiriéndose a México) no tiene la autoridad moral para hablar de Venezuela».

\section{Reflexiones finales}

Lo anterior, más allá de retratar la singularidad en los niveles de aproximación que se han venido gestando a últimas fechas a partir de la tensión generada en las relaciones México-Estados Unidos tras el ascenso de Donald Trump al poder, lo que deja al descubierto este análisis es que, en medio de esta mutua e histórica desconfianza que reina entre ambas naciones; se privilegian esquemas de acercamiento muy originales, los cuales corresponden, en gran medida, al trabajo de aproximación y consensos que vienen adelantando, principalmente las secretarías dedicadas a los asuntos internos y externos (Cancillería), las jefaturas de comercio y hacienda, al tiempo las que concentran los temas de defensa y seguridad tanto en México como en Washington.

Ahora bien, desde la perspectiva que marca la llegada al poder en Estados Unidos del candidato republicano Donald Trump, se inicia una era que, más allá de las particularidades mediáticas y las expectativas generadas por este empresario, lo que en realidad evidencia es un momentum clave en el reposicionamiento global de Estados Unidos. Lo que desde la perspectiva de Trump implica, por un lado, entretejer los lineamientos del nacionalismo que enmarca a través de su discurso del America First, y por el otro, adicionar una carga de proteccionismo económico e industrial a partir del cual se refuerce las bondades de lo que en las condiciones actuales de Estados Unidos, significa impulsar un esquema de gobierno basado en la idea de «corporatocracia».

Con respecto a México, cabe señalar que la llegada del presidente Trump a la Casa Blanca, a pesar de haber generado un ambiente de enorme tensión e incertidumbre que se apreció con rigurosidad el propio día de la elección que lo que se conoce en Estados Unidos como el Super Martes, cuando la divisa mexicana fluctuaba vertiginosamente entre los 18 pesos hasta pretender alcanzar el tope de los 25 pesos, que en realidad no se alcanzó, pero que generó la mayor de las expectativas entre los inversionistas en México, terminaría por despertar lo que en términos de la opinión pública y de algunos sectores opositores al gobierno del presidente Enrique Peńa Nieto, poco se esperaba antes de iniciarse el fenómeno Trump: el despertar de la unidad nacional. 
Bajo este despertar de la unidad nacional, algo tardío en la actual administración de Peña Nieto, según la opinión de algunos analistas en México; ciertamente ese ha sido el factor primordial, el motor decisivo para impulsar las decisiones de Estado que, como se describió en este trabajo, han permitido solventar y trascender de los mensajes de Twitter entre Trump y Peña, y relanzar un diálogo abierto y fluido entre las instituciones homólogas entre los dos países.

Asimismo, México ha recibido el aval para convertirse en ese «actor con responsabilidad global», encargado de atender asuntos propios del continente. Lo observamos en su acompañamiento en las tareas asignadas para generar el desarrollo esperado en el área geoestratégica del Triángulo Norte, pero también de lo que vendría a ser una declaración frontal de alineamiento de la actual Venezuela de Nicolás Maduro, con los principios fundamentales que exige la consolidación de una democracia en nuestros tiempos. Siendo este último caso, el de las relaciones México-Venezuela, un tema ríspido en materia de política exterior mexicana, con profundas repercusiones en los asuntos domésticos, tomando en consideración la próxima contienda por la silla presidencial en 2018, a la que se postula con notable apoyo social el exregente de la Ciudad de México, Andrés Manuel López Obrador, cuya postura ideológica resulta favorable a los criterios políticos que experimenta la Venezuela actual a su paso por lo que en su momento Chávez concibió como la entrada al «Socialismo del siglo XXI».

\section{Referencias}

Alcántara Silva, P. G. (2017, 28 de febrero). Expectativas ante las politicas del presidente estadounidense Donald Trump. México: Instituto de Investigaciones Estratégicas de la Secretaria de Marina (Ininvestam). Recuperado de www.cesnav.gob.mx

BBC Mundo (2016a, 15 de marzo). Las verdaderas cifras de los hispanos en EE.UU. y cuánto poder tienen. Recuperado de http://www.bbc.com/mundo/noticias/2016/03/ 160304_internacional_elecciones_eeuu_2016_cifras_latinos_lf

BBC Mundo (2016b, 13 de noviembre). Estados Unidos, Donald Trump dice que deportará hasta 3 millones de indocumentados con antecedentes judiciales. Recuperado de http:// www.bbc.com/mundo/noticias-internacional-37966905

BBC Mundo (2017a, 1 marzo). «Un nuevo capitulo de la grandeza de Estados Unidos»: Donald Trump sorprende con el optimismo de su discurso ante el Congreso. Recuperado de http:// www.bbc.com/mundo/noticias-39124200

BBC Mundo (2017b, 26 de enero). Enrique Peña Nieto y Donald Trump cancelan su reunión en la Casa Blanca por polémica sobre el muro entre Estados Unidos y México. Recuperado de http://www.bbc.com/mundo/noticias-america-latina-38763941

García, C. (2017). Inicia OEA en Cancún su 47 Asamblea. Periódico El Universal, 19 de junio. 
González, Y. (2017). La verdad detrás del déficit comercial de EU con México. Revista Expansión en alianza con CNN, 26 de enero. Recuperado de http://expansion.mx/ economia/2017/01/26/la-verdad-detras-del-deficit-comercial-de-eu-con-mexico

Hernández, E. (2017). Esta es la propuesta de Slim para negociar con Trump y salvar a México. Recuperado de www.forbes.com.mx, 28 de enero.

Hernández Borbolla, M. (2017). La alianza militar con la que EU busca que México «haga el trabajo sucio» en Centroamérica. Recuperado de www.huffingtonpost.com.mx, 21 de marzo.

Palm, S. J. y Stikkers, K. W. (2017). El sueño americano de Claire Underwood: ¿qué dejaremos al morir? En W. Irwin y E. Hackett, House of Cards y la filosofía. La república de Underwood. México: Rica Editorial.

Pastor Gómez, M.L. (2016). México: entre el muro de la frontera norte y la porosidad de la frontera sur. Madrid: Instituto Español de Estudios Estratégicos. Recuperado de www. cesnav.edu.mx/ININVESTAM/docs/docs_analisis/da_27-16.pdf

Presidencia de la República (2016). Relación México-Estados Unidos, www.gob.mx, 21 de julio.

Trump, D. (2015). Crippled America. How to make America Great Again. Nueva York: Threshold Editions.

\section{Páginas electrónicas}

www.whitehouse.gov

www.nodal.am

www.elhorizonte.mx

www.gob.mx

Fecha de recepción: 20 de mayo de 2017

Fecha de aprobación: 25 de junio de 2017 